\title{
Synergistic Effect of a Prolonged Combination Course of Tranexamic Acid and Dexamethasone Involving High Initial Doses in Total Knee Arthroplasty: A Randomized Controlled Trial
}

\author{
Hong $\mathrm{Xu}, \mathrm{MD}^{1, *}$ Jinwei $\mathrm{Xie}, \mathrm{MD}^{1, *} \quad$ jingli Yang, $\mathrm{PhD}^{2, *}$ \\ Fuxing Pei, $\mathrm{MD}^{3}$
${ }^{1}$ Department of Orthopaedic Surgery and National Clinical Research Center for Geriatrics, West China Hospital, Sichuan University, Sichuan, China
${ }^{2}$ College of Earth and Environmental Sciences and School of Public Health, Lanzhou University, Lanzhou, Gansu, China
3 Department of Orthopaedic Surgery, West China Hospital, Sichuan University, Sichuan, China

Zeyu Huang, $\mathrm{MD}^{3}$

Duan Wang, $\mathrm{MD}^{3}$

J Knee Surg 2023;36:515-523.

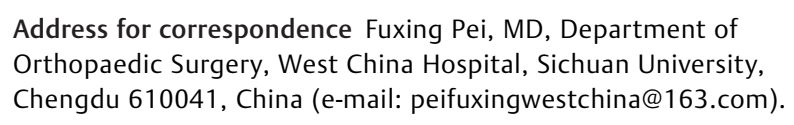

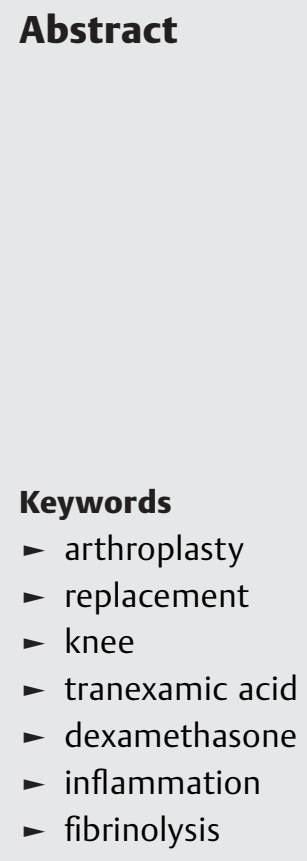

The optimal regimes of tranexamic acid (TXA) and dexamethasone (DXM) in total knee arthroplasty (TKA) are still uncertain. The aim of this study was to assess the efficacy and safety of a prolonged course of intravenous TXA and DXM involving a high initial dose in TKA. Patients who underwent primary TKA at our center were randomized to receive one of four regimes: control (group $A$ ), prolonged course of TXA (B), prolonged course of DXM (C), or the combination of a prolonged course of TXA and DXM (D). The four groups were compared in primary outcomes (fibrinolytic and inflammatory markers, knee function, postoperative pain levels, and consumption of opioids) and secondary outcomes (blood loss, maximal drop in hemoglobin, coagulation, fasting blood glucose, and complications). A total of 162 patients were enrolled. On postoperative days 2 and 3, fibrinolytic markers were lower in groups $B$ and $D$ than in groups $A$ and $C$; inflammatory markers were lower in groups $C$ and $D$ than in groups $A$ and $B$. Inflammatory markers were lower in group $B$ than in group $A$ on postoperative day 3. Postoperative pain levels and oxycodone consumption were lower, and knee function was better in groups $C$ and D. The four groups did not differ in any of the secondary outcomes. A prolonged course of intravenous TXA and DXM involving high initial doses can effectively inhibit postoperative fibrinolytic and inflammatory responses, reduce pain, and improve knee function after TKA.
Total knee arthroplasty (TKA) is a successful surgical treatment for severe end-stage knee diseases. However, hyper-

\footnotetext{
Hong $\mathrm{X} u$, Jinwei Xie, and Jingli Yang are co-first authors. They equally contributed to this article.
}

fibrinolysis due to surgical trauma causes blood loss that may require transfusion and can prolong postoperative recovery. ${ }^{1}$ Administration of tranexamic acid (TXA), a lysine analogue that acts as a competitive inhibitor of plasmin(-ogen), can block the fibrinolytic response, significantly reducing blood received

January 7, 2021

accepted after revision

September 21, 2021

article published online

November 18, 2021 (c) 2021. Thieme. All rights reserved.

Thieme Medical Publishers, Inc., 333 Seventh Avenue, 18th Floor, New York, NY 10001, USA
DOI https://doi.org/ 10.1055/s-0041-1739197. ISSN 1538-8506. 
loss and the need for transfusion. ${ }^{2}$ However, the optimal TXA regime is still uncertain. Postoperative hidden blood loss (HBL) can last for 3 days after TKA, and administering TXA 3-day postoperatively can reduce total blood loss (TBL) as well as mitigate hemoglobin drop, inflammatory response, and fibrinolysis. ${ }^{3}$ However, most current guidelines recommend using TXA only within 24 hours after TKA. ${ }^{4}$ The potential benefits of prolonged TXA administration with a high initial dose should therefore be assessed rigorously in TKA.

Excessive inflammation caused by surgical stress can cause many adverse experiences and outcomes to patients including postoperative nausea and vomiting (PONV), pain, and fatigue as well as more serious adverse events such as angina pectoris and gastrointestinal bleeding. ${ }^{5}$ Satisfactory control of postoperative inflammation is crucial for promoting recovery. Dexamethasone (DXM), a long-acting glucocorticoid with a powerful anti-inflammatory effect, has been widely used to inhibit postoperative inflammatory response in TKA. A single, low dose of DXM (5-10 mg) can reduce PONV incidence and pain at 24 hours but not 48 hours after TKA. ${ }^{6}$ Single-dose DXM has a half-life around 36 to 55 hours, and its effect is obvious within 24 to 48 hours after TKA, ${ }^{7}$ yet previous published work showed that the inflammatory markers $C$-reaction protein (CRP) and interleukin-6 (IL-6) can remain elevated as long as 72 or even 96 hours after TKA. ${ }^{8}$ Thus, single-dose DXM does not control inflammation or pain adequately at 48 or 72 hours after surgery. ${ }^{7}$ These considerations highlight the need to assess the efficacy of prolonged DXM administration in TKA. ${ }^{9}$

The combined use of TXA and DXM may help reduce inflammation and relieve pain in TKA. ${ }^{10} \mathrm{~A}$ previous study showed that a high initial TXA dose $(60 \mathrm{mg} / \mathrm{kg})$ followed by five TXA doses within 24 hour postoperatively combined with $20 \mathrm{mg}$ DXM during anesthesia induction intravenously was effective for reducing blood loss, inhibiting fibrinolytic and inflammatory responses. ${ }^{11}$ However, the control of pain as well as fibrinolytic and inflammatory responses was unsatisfactory at 24 hours after surgery.

Hence, the present randomized controlled study was conducted to assess (1) whether a prolonged TXA course involving a high initial dose can inhibit fibrinolysis and inflammation more than the "control" treatment that was previously examined, ${ }^{11}$ without altering the incidence of venous thromboembolism (VTE) or other serious complications; (2) whether a prolonged DXM course involving a high initial dose can reduce postoperative inflammation, pain and opioid use more than the control treatment; and (3) whether the combination of the prolonged course of TXA and DXM can inhibit fibrinolysis and inflammation more effectively in TKA, without increasing the risk of complications.

\section{Methods}

\section{Study Design and Patients}

The protocol of this randomized controlled trial was approved by our institutional review board and registered in the Chinese Clinical Trial Registry (ChiCTR1900026092). Written informed consent was obtained from each patient before enrollment.
Patients who underwent primary unilateral TKA for osteoarthritis between September 2019 and August 2020 were screened for enrollment. Patients were excluded from the study if their TKA was a revision or bilateral, if they were younger than 18 years or older than 80 years, if they were allergic to TXA or DXM, or if they were using anticoagulants, had a history of myocardial infarction, angina, stroke, severe renal or liver failure, deep vein thrombosis (DVT), pulmonary embolism (PE), or inflammatory disease. Enrolled patients were randomized by computer into one of four groups.

Group A (control group) received $60 \mathrm{mg} / \mathrm{kg}$ TXA intravenously at 5 to 10 minutes before skin incision, followed by five doses of $1 \mathrm{~g}$ TXA at $3,6,12,18$, and 24 hours after the initial dose. This group also received $20 \mathrm{mg}$ DXM intravenously during induction of anesthesia. Group $B$ received the same treatment as group A, as well as three doses of $1 \mathrm{~g}$ TXA at 36, 48, and 60 hours after the initial dose. Group $C$ received the same treatment as group A, as well as $10 \mathrm{mg}$ DXM at 08:00 to 09:00 hour on postoperative day (POD) 1 and $5 \mathrm{mg}$ DXM at the same time on POD 2. Group D received all the treatments given to groups $B$ and C. DXM was given at 08:00-09:00 hour to groups $C$ to $D$, because giving the drug at the nadir of the patient's secretion of endogenous adrenocortical hormone may inhibit adrenocortical function the next day.

Saline was given as placebo for DXM and TXA infusions in this study. Patients, surgeons, data collectors, and analysts remained blinded to patient allocation throughout the study, whereas the attending physician was told of the allocation after surgery.

\section{Surgical Procedure}

All surgical procedures were performed by a senior surgeon while the patient was under general anesthesia. In all cases, a standard midline skin incision, medial parapatellar approach, and measured resection technique were used with a cemented posterior-stabilized prosthesis. All procedures were performed without a tourniquet, drainage or autotransfusion system. Before the wound was closed, $200 \mathrm{mg}$ ropivacaine in $60 \mathrm{~mL}$ normal saline was injected into the periarticular space for all patients.

\section{Postoperative Care}

Patients were routinely managed according to a standardized clinical pathway. Enoxaparin (2000 IU) was injected subcutaneously at 6 to 8 hours postoperatively, then once every 24 hours until discharge, and rivaroxaban $(10 \mathrm{mg}$ ) was followed orally once daily at the same time of each day after discharge, during 2 weeks. Diclofenac (50 mg, q12h) was given orally to all patients to relieve postoperative pain. Oxycodone $\mathrm{HCl}$ sustained-release tablet $(10 \mathrm{mg}$ ) was administered if the patient reported pain $\geq 4$ on a visual analog scale (VAS; $0=$ no pain, $1-3=$ mild pain, $4-6=$ moderate pain, $7-10=$ severe pain); morphine $(5 \mathrm{mg})$ was injected intramuscularly if patients reported pain $\geq 7$ on the VAS. If the patient developed severe nausea or vomiting, ondansetron (4 mg) was injected intravenously. All patients had the same postoperatively mobilization plan. On the day of surgery and first day after the surgery, only straightening 
exercise was performed, and flexion began on the second day after the surgery

Transfusion was performed if the patient's condition met the criteria established by the Chinese National Ministry of Health $^{2}$ : (1) if hemoglobin level was $<70 \mathrm{~g} / \mathrm{L}$; (2) or if hemoglobin level was between 70 and $100 \mathrm{~g} / \mathrm{L}$ and the patient showed dizziness, palpitations, fatigue, or other obvious symptoms of anemia. All patients were followed up for 3 months after surgery.

\section{Outcomes}

Data were recorded on patient demographics, preoperative characteristics, and comorbidities. The primary outcomes included fibrinolytic and inflammatory markers, knee function, postoperative pain levels, and consumption of opioids, while the secondary outcomes included blood loss, maximal drop in hemoglobin, coagulation, fasting blood glucose (FBG), and complications.

The following variables were measured perioperatively: levels of plasma fibrinolysis markers [fibrin(-ogen) degradation products (FDP) and D-dimer], levels of serum CRP and IL-6, and levels of coagulation markers [prothrombin (PT), activated partial thromboplastin time (APTT), fibrinogen, and international normalized ratio (INR) and platelet count.

Pain level was evaluated using the above-mentioned VAS before surgery, at 15:00 to 17:00 hour on POD 1 to 3 , at 2 to 3 weeks, and at 3 months. Numbers of patients needing analgesic rescue were recorded, as was the consumption of opioids. Range of motion (ROM), defined as the sum of the flexion and extension degrees, was measured in the operated knee before surgery, on POD 3, at 2 to 3 weeks and at 3 months.

Patient's blood volume was calculated using the Nadler Eq, ${ }^{12}$ while TBL was evaluated using the Gross Eq. ${ }^{13}$ Intraoperative blood loss (IBL) was determined by adding the weight of blood-soaked gauzes and compresses to the volume aspirated from the surgical field, then subtracting the dry weight of the gauzes and compresses as well as the volume used to wash the surgical field during the procedure. ${ }^{14}$ HBL was calculated as the difference between TBL and IBL. ${ }^{15}$

FBG was recorded in the morning during hospitalization. DVT was identified based on clinical symptoms and Doppler ultrasonography, which was conducted before surgery, on the day of discharge, as well as at 2 to 3 weeks and 3 months postoperatively.

\section{Data Treatment and Statistical Analysis}

Sample size was estimated using an online sample size calculator (http://powerandsamplesize.com/) and data from previous published study. ${ }^{11}$ We chose one of the primary outcomes, CRP, to estimate the sample size due to it can reflect the effect of TXA and DXM simultaneously; moreover, its level is strongly associated with postoperative pain, knee function, and patient satisfaction. ${ }^{16}$ These calculations determined that at least 33 patients were required in each group to detect CRP reduction of $15 \mathrm{mg} / \mathrm{L}$ on POD 3 with a power of 0.80 at a significance level of 0.05 . Assuming a $20 \%$ dropout rate, we aimed to recruit at least 40 in each study arm.

Measurements under the manufacturer-specified limit of detection were re-expressed as the "lower limit/2."17 For example, FDP levels below $2.5 \mathrm{mg} / \mathrm{L}$ were re-expressed as $1.25 \mathrm{mg} / \mathrm{L}$; IL-6 levels below $1.5 \mathrm{pg} / \mathrm{mL}$ were expressed as $0.75 \mathrm{pg} / \mathrm{mL}$.

Quantitative data were reported as mean \pm standard deviation, while qualitative data were reported as frequencies or ratios. Differences among groups were assessed for significance using one-way analysis of variance in the case of quantitative variables or the chi-squared test in the case of qualitative variables. All data were analyzed using SPSS 21 (IBM, Armonk, NY) and a significance definition of $p<0.05$. Graphs were generated using GraphPad Prism 8.30 (GraphPad Software, San Diego, CA).

\section{Results}

A total of 187 patients who underwent primary unilateral TKA for osteoarthritis between September 2019 and August 2020 were screened for enrollment. After excluding 15 patients who met any of the exclusion criteria and another 4 who declined to participate, we included 162 patients in the final analysis (-Supplementary Fig. S1, available in the online version only). The four treatment groups did not differ significantly in demographics, comorbidities, or surgical parameters (-Table $\mathbf{1}$ ).

\section{Fibrinolysis, Coagulation, and Platelet Count}

FDP and D-dimer levels did not differ significantly across the four groups on POD 1 or 15. On POD 2 and 3, FDP and D-dimer levels were significantly lower in groups B and D than in groups A and C (-Fig. 1). Mean values for PT, APTT, INR, fibrinogen, and platelet count were within the normal range during the perioperative period and did not differ significantly across the four groups (-Supplementary Figs. S2 and s3, available in the online version only).

\section{Inflammatory Markers}

CRP and IL-6 levels did not differ significantly among the four groups before surgery or on POD 1 or 15. On POD 2, levels of both markers were significantly lower in groups $C$ and $D$ than in groups A and B. On POD 3, CRP levels were significantly higher in group $A$ than in the other groups; and CRP was significantly higher in group B than in group D; IL-6 level was significantly lower in groups $C$ and $D$ than in groups $A$ and $B$. Marker levels were lower in group B than in group A ( - Fig. 2).

\section{Pain Level and Analgesic Rescue}

The four groups did not differ significantly in VAS scores for dynamic or rest pain before surgery or on POD 15 (-Fig. 3). In fact, rest pain levels did not differ significantly among the groups throughout the perioperative period. In contrast, on POD 1 to 3, groups C and D reported significantly lower dynamic pain than the other groups.

Consistent with the observed differences in dynamic pain, groups A and B contained higher proportions of patients 


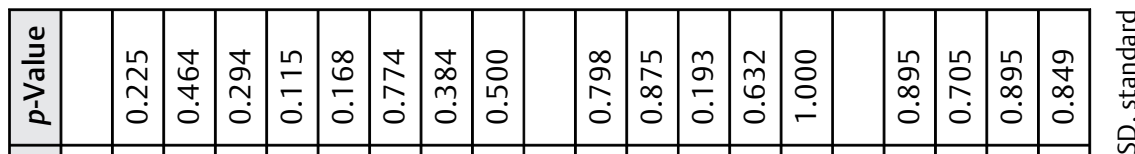

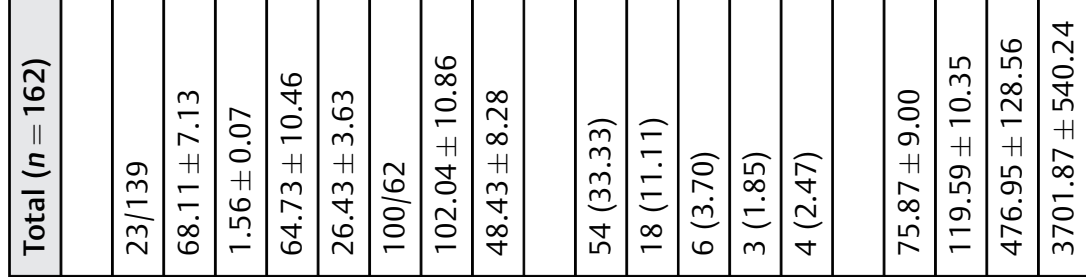

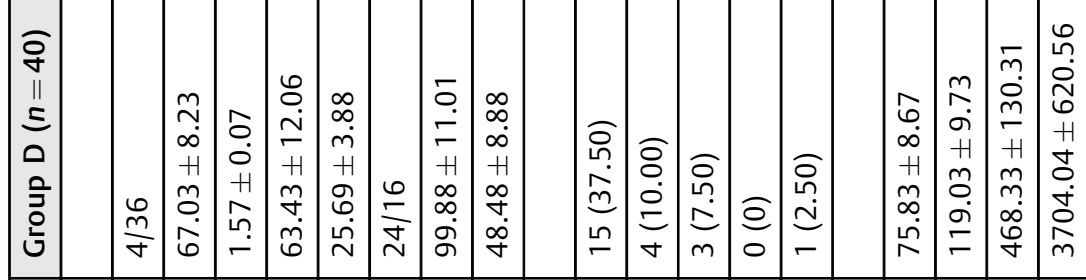

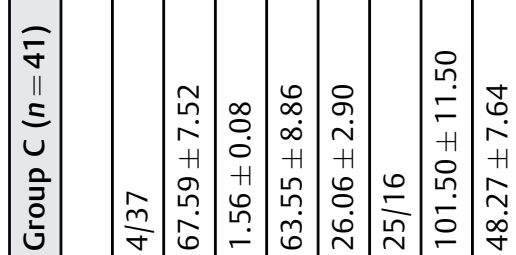

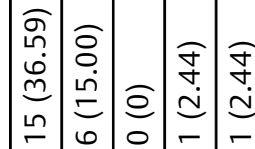

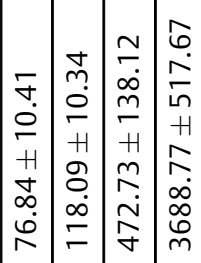

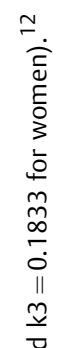

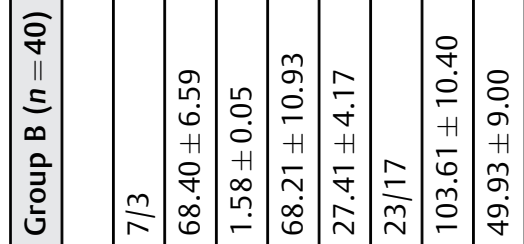

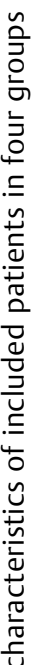

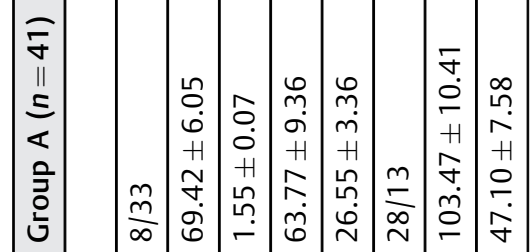

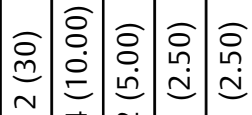

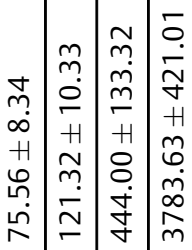

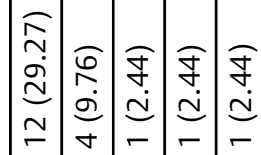

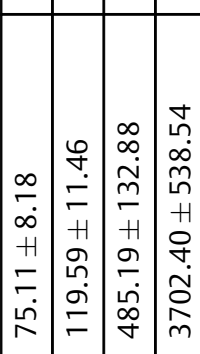

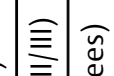



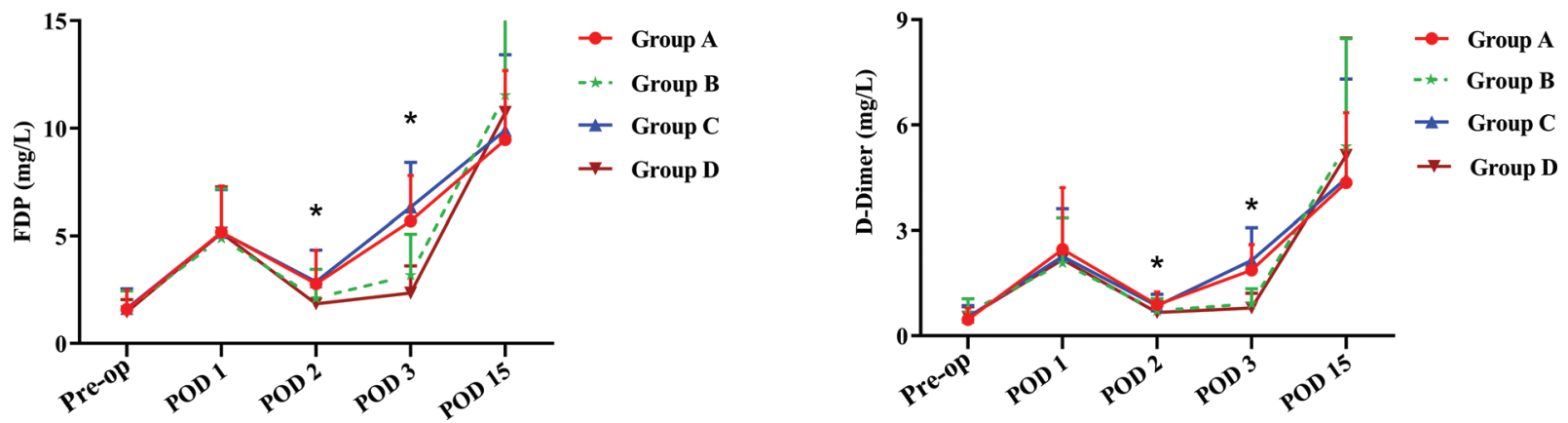

Fig. 1 Levels of fibrin degradation products (FDP) and D-dimer in the four groups. Pre-op: preoperative day; POD, postoperative day. ${ }^{*} p<0.05$.
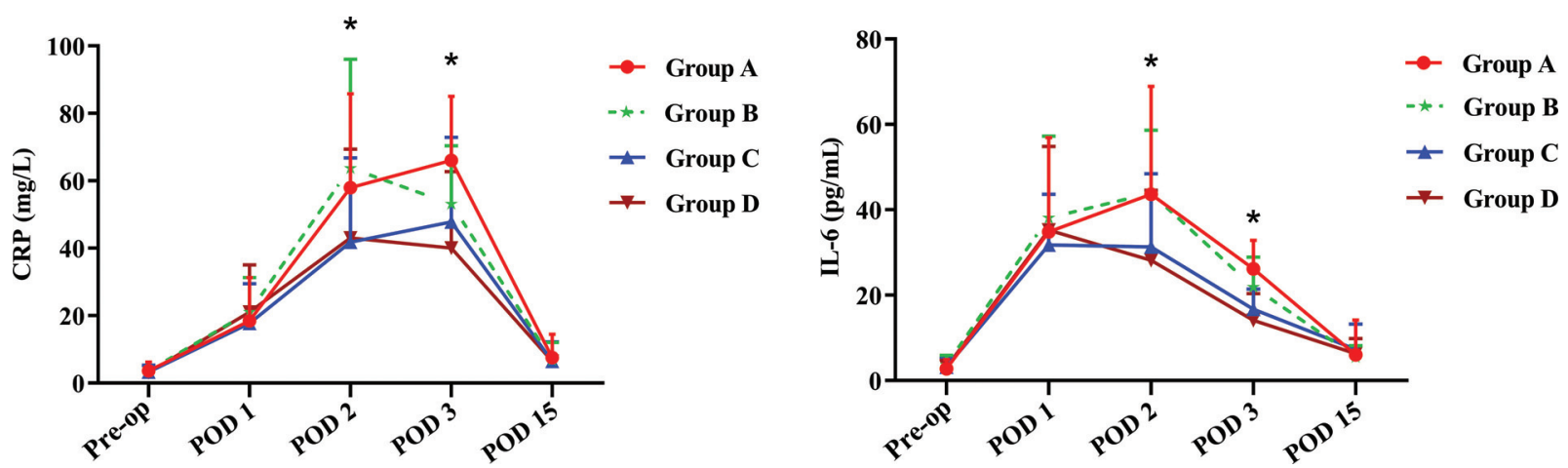

Fig. 2 Levels of C-reactive protein (CRP) and interleukin-6 (IL-6) in the four groups. Pre-op, preoperative day; POD, postoperative day. ${ }^{*} p<0.05$.
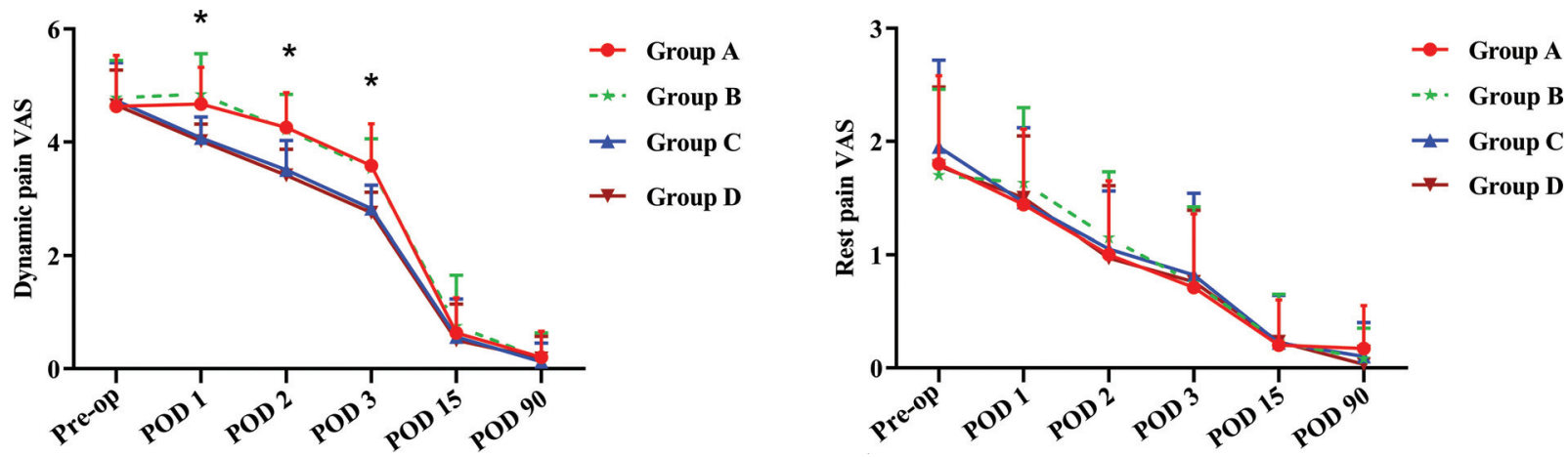

Fig. 3 Levels of pain on a visual analog scale (VAS) in the four groups. Pre-op, preoperative day; POD, postoperative day. ${ }^{*} p<0.05$.

requiring analgesic rescue with oxycodone and they consumed more of the drug than groups $C$ and D on POD 1 and throughout the hospital stay. However, no such differences were observed on the day of surgery or on POD 2 or 3 (-Table 2 ). The four groups did not differ significantly in the number of patients requiring morphine or in the amount of morphine consumed on the day of surgery, on POD 1 to 3 or throughout the rest of postoperative hospitalization (-Supplementary Table S1 available in the online version only).

\section{Blood Loss, Transfusion, and Postoperative Length of Stay}

The four groups did not differ significantly in TBL, IBL, HBL, maximal hemoglobin drop, or postoperative length of stay (-Supplementary Table S2, available in the online version only). None of the patients required transfusion.

\section{Functional Assessment and FBG}

While flexion function and ROM of the operated knee were similar among the four groups before surgery and on POD 90, these outcomes were significantly greater in groups $C$ and D on POD 3 and 15 (-Fig. 4). In all four groups, FBG was significantly higher on POD 1 than before surgery. However, the four groups showed no significant differences in FBG before surgery or on POD 1-3 (-Supplementary Fig. S4, available in the online version only).

\section{PONV, Anti-Emetic Use, VTE, and Complications}

The four groups did not differ significantly in PONV incidence or ondansetron consumption on the day of surgery, POD 1 to 3 or anytime during postoperative hospitalization (-Supplementary Table S3, available in the online version only). Similarly, the four groups did not differ significantly in 
Table 2 Use of oxycodone in the four groups

\begin{tabular}{|c|c|c|c|c|c|}
\hline Variable & Group A $(n=41)$ & Group B $(n=40)$ & Group C $(n=41)$ & Group D $(n=40)$ & $p$-Value \\
\hline \multicolumn{6}{|l|}{ Total } \\
\hline$n(\%)$ & $8(19.51)$ & $7(17.50)$ & $2(4.88)$ & $1(12.50)$ & $0.018^{\mathrm{a}}$ \\
\hline Dosage $(\mathrm{mg})$ & 130 & 110 & 20 & 10 & $0.011^{\mathrm{a}}$ \\
\hline \multicolumn{6}{|l|}{ Operative day } \\
\hline$n(\%)$ & $1(2.44)$ & $1(2.50)$ & $1(2.44)$ & 0 & 0.632 \\
\hline Dosage $(\mathrm{mg})$ & 10 & 20 & 10 & 0 & 0.563 \\
\hline \multicolumn{6}{|c|}{ Postoperative day 1} \\
\hline$n(\%)$ & 7 (17.07) & $6(15.00)$ & $1(2.44)$ & $1(2.50)$ & $0.019^{\mathrm{a}}$ \\
\hline Dosage $(\mathrm{mg})$ & 100 & 90 & 10 & 10 & $0.037^{\mathrm{a}}$ \\
\hline \multicolumn{6}{|c|}{ Postoperative day 2} \\
\hline$n(\%)$ & $1(2.44)$ & 0 & 0 & 0 & 0.429 \\
\hline Dosage $(\mathrm{mg})$ & 20 & 0 & 0 & 0 & 0.402 \\
\hline \multicolumn{6}{|c|}{ Postoperative day 3} \\
\hline$n(\%)$ & 0 & 0 & 0 & 0 & - \\
\hline Dosage $(\mathrm{mg})$ & 0 & 0 & 0 & 0 & - \\
\hline
\end{tabular}

${ }^{\mathrm{a}} \mathrm{p}<0.05$.
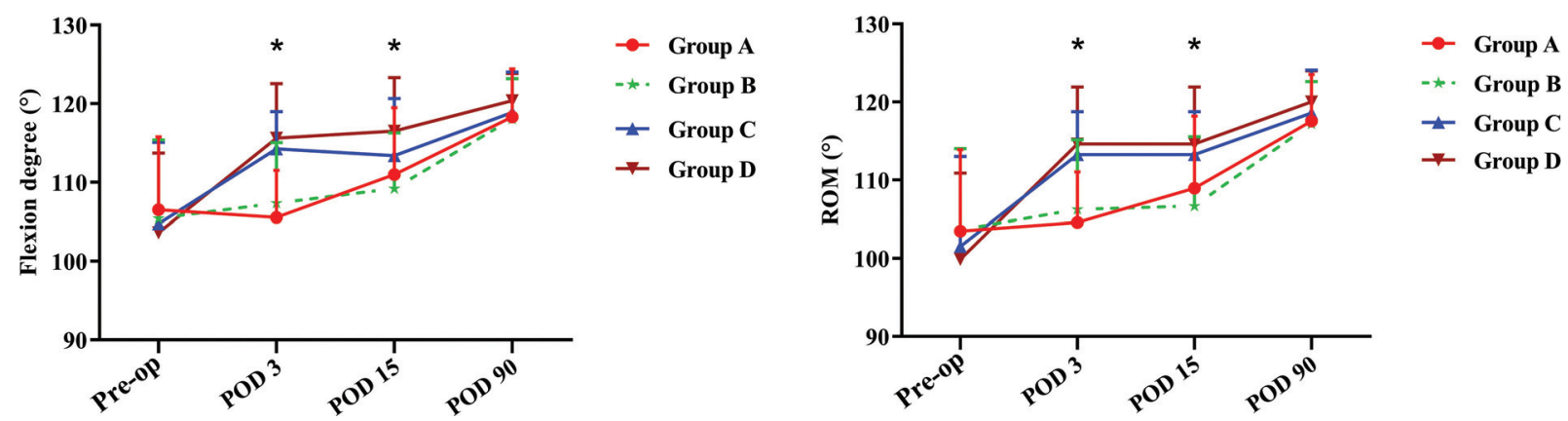

Fig. 4 Flexion function and range of motion (ROM) in the four groups. Pre-op, preoperative day; POD, postoperative day. ${ }^{*} p<0.05$.

incidence of intermuscular vein thrombosis or VTE before surgery or on POD 3, 15, or 90 (-Supplementary Table S4, available in the online version only). One patient in group $A$ and one in group B were diagnosed with a DVT on POD 15 on an outpatient basis. Both patients showed DVT in the posterior tibial vein without obvious clinical manifestations. No severe complications were observed during hospitalization or follow-up, which included no PE, seizures, superficial or periprosthetic joint infection, myocardial and cerebral infarction, acute gastrointestinal hemorrhage, unplanned readmission, or mortality.

\section{Discussion}

Although many studies have confirmed the safety and efficacy of TXA and DXM in TKA, optimal regimes are still controversial. ${ }^{18,19}$ In addition, few studies have examined their simultaneous use, which is an interesting question because the two drugs overlap in some of their bioactivities, for example, both have anti-inflammatory effects. The present study was, therefore, conducted that suggests that a prolonged course of TXA and DXM combination therapy involving initially high doses can inhibit post-TKA fibrinolytic and inflammatory responses, relieve postoperative pain, and improve knee function to a greater extent than the combination regime that was previously examined. ${ }^{11}$

One study ${ }^{20}$ reported that fibrinolysis peaked at 6 hours after TKA and persisted until $\sim 18$ hour, but other work suggests that the fibrinolytic markers FDP and D-dimer can remain elevated even until POD 3. ${ }^{21,22} \mathrm{HBL}$ can also persist until POD 3, which may be associated with hyperfibrinolysis and functional exercise of the operated knee at an early stage. ${ }^{23}$ Therefore, we examined whether prolonging the use of TXA to 60 hour postoperatively might provide additional benefit. We found that a high initial TXA dose followed by five additional low doses within 24 hours after surgery led to similar TBL as a 3-day TXA course, even though the prolonged course inhibited fibrinolysis through POD 3. Given that another study found that a 3-day low-dose TXA course led to lower TBL and smaller maximal hemoglobin drop than a single preoperative TXA dose, ${ }^{3}$ we hypothesize that a high initial TXA dose followed by five additional low 
doses within 24 hours after TKA can effectively control blood loss.

Aseptic inflammation caused by surgical trauma is a defensive response that allows the body to adapt quickly to stress, promote tissue regeneration and wound healing, and clear pathogens. ${ }^{5}$ However, excessive inflammation caused by osteotomy and soft tissue incision during TKA can trigger postoperative PONV, pain, and fatigue. ${ }^{24}$ Indeed, levels of the inflammatory markers CRP and IL-6 are related to postoperative pain, knee function, and patient satisfaction. ${ }^{16,25} \mathrm{Sev}-$ eral studies have demonstrated the ability of a low dose of DXM on its own to relieve postoperative pain for up to 24 hours. ${ }^{6,26}$ One study found that $16 \mathrm{mg}$ DXM administered preoperatively could halve CRP on POD 3, but this still meant high levels of $90 \mathrm{mg} / \mathrm{L}$ on POD 3 and $70 \mathrm{mg} / \mathrm{L}$ on POD $5 .{ }^{27}$ In the present study, a prolonged course of TXA and DXM combination therapy involving initially high doses significantly decreased CRP to $(47.83 \pm 25.07) \mathrm{mg} / \mathrm{L}$ and IL-6 to $(16.67 \pm 4.75) \mathrm{mg} / \mathrm{L}$ on POD3. These results suggest that TXA can supplement the powerful anti-inflammatory effects of DXM to further decrease the inflammatory response on POD 3. TXA can decrease inflammation by inhibiting fibrinolysis, which creates plasmin that stimulates inflammatory cells and activates the complement system. ${ }^{28}$ Better control of inflammation in group D is reflected in the smaller proportion of patients requiring oxycodone, lower consumption of oxycodone, and better knee function.

Given its strong inhibition of fibrinolysis, TXA has the potential to affect normal coagulation. This is a particular concern when the drug is repeatedly administered at high doses. However, none of our treatment regimens significantly affected coagulation function. Previous study ${ }^{11}$ revealed that a high initial dose followed by five doses of TXA had no obvious effect on coagulation in TKA, consistent with other studies. ${ }^{29}$ In addition, plasmin can cause platelet dysfunction by inducing the degradation of platelet glycoprotein and activating the complement pathway. ${ }^{30,31}$ DXM can prevent activated macrophages from inhibiting platelet aggregation. ${ }^{32}$ Interestingly, a prolonged course of TXA can inhibit plasma activation, thereby protecting platelet function. ${ }^{33}$

By inducing hepatic gluconeogenesis and increasing insulin resistance, DXM has the potential to raise blood glucose levels, which leads some clinicians to worry about increased risk of wound complications and infection. ${ }^{34,35}$ We found that administering $20 \mathrm{mg}$ DXM intravenously during anesthesia induction slightly increased FBG on POD 1 but not on later days. Administering two low doses of DXM on POD 1 to 2 did not significantly increase FBG on POD 2 to 3 . Our results are consistent with previous work suggesting that DXM is less likely than the surgical trauma itself to trigger postoperative hyperglycemia. 36,37

In addition to the differences in inflammatory and fibrinolytic markers, we found that the prolonged use of TXA and DXM can significantly relieve early postoperative pain, reduce use of the opioid analgesic, oxycodone, and improve early knee function. That is particularly important to reduce the incidence of postoperative chronic pain, ${ }^{38}$ decrease opioid dependence, improve patient experience, increase patient satisfaction and the safety of the operation. ${ }^{39}$ Hence, although the knee function, pain level, and rate of complications did not differ at 3 months postoperatively, we supposed that patients could benefit from the prolonged use of TXA and DXM.

Our results should be interpreted with caution given some limitations. First, the limited sample means that larger studies involving longer follow-up are needed to draw definitive conclusions about the safety of a prolonged course of TXA and DXM combination therapy involving initially high doses. Second, future studies should examine whether TXA and DXM delay wound healing, since both fibrinolysis and inflammatory processes are inhibited and they play an important role in wound healing. ${ }^{40,41}$ Third, the optimal degree of postoperative fibrinolysis and inflammatory response should be further explored. Finally, no patients at high risk for developing DVT/PE were included, only general anesthesia was used and this may not apply to those patients undergoing regional anesthesia, and the study was not powered for detection of adverse events such as DVT/PE.

\section{Conclusions}

Our study suggests that the combined use of a prolonged course of TXA and DXM can inhibit postoperative fibrinolytic and inflammatory response, relieve postoperative pain, and improve knee function after TKA within the first few postoperative days. No differences in complications, pain, or ROM were demonstrated at 90 days postoperatively. Further investigation is necessary to elucidate the long-term effect of prolonged TXA and DXM usage following TKA, and whether or not the early differences in serum markers, ROM, and pain relief play a role in long-term success of TKA surgery.

Note

This study was prospectively registered in the Chinese Clinical Trial Registry (ChiCTR1900026092).

\section{Ethical Approval}

The study protocol was approved by clinical trials and biomedical ethics committee, West China Hospital, Sichuan University.

\section{Authors' Contributions}

H.X. and J.W. X. performed the study design, data collection, writing and revision of the manuscript. J.L. Y. and Z.Y. H. analyzed and interpreted data for the work. D.W. participated in final approval of the version to be published. F.X. P. contributed to the conception and design of the work and revised the manuscript. All authors read and approved the final manuscript.

\section{Funding}

The research was supported by Post-Doctor Research Project, West China Hospital, Sichuan University (2018HXBH073); National Clinical Research Center for Geriatrics, West China Hospital, Sichuan University (Z2018B11). 
Conflict of Interest

None declared.

\section{Acknowledgments}

The authors thank all patients, surgeons, and nursing staffs involved in this study for their support during study period. We also thank A. Chapin Rodríguez, PhD, from Creaducate Enterprises Ltd for editing the English text of a draft of this manuscript.

\section{References}

1 Burleson A, Guler N, Banos A, et al. Perioperative factors and their effect on the fibrinolytic system in arthroplasty patients. Clin Appl Thromb Hemost 2016;22(03):274-279

2 Xie J, Ma J, Yao H, Yue C, Pei F. Multiple boluses of intravenous tranexamic acid to reduce hidden blood loss after primary total knee arthroplasty without tourniquet: a randomized clinical trial. J Arthroplasty 2016;31(11):2458-2464

3 Wu X-D, Tian M, He Y, et al. Efficacy of a three-day prolongedcourse of multiple-dose versus a single-dose of tranexamic acid in total hip and knee arthroplasty. Ann Transl Med 2020;8(06): 307-307

4 Fillingham YA, Ramkumar DB, Jevsevar DS, et al. The safety of tranexamic acid in total joint arthroplasty: a direct meta-analysis. J Arthroplasty 2018;33(10):3070-3082.e1

5 Desborough JP. The stress response to trauma and surgery. $\mathrm{Br} J$ Anaesth 2000;85(01):109-117

6 Koh IJ, Chang CB, Lee JH, Jeon YT, Kim TK. Preemptive low-dose dexamethasone reduces postoperative emesis and pain after TKA: a randomized controlled study. Clin Orthop Relat Res 2013;471 (09):3010-3020

7 Samona J, Cook C, Krupa K, et al. Effect of intraoperative dexamethasone on pain scores and narcotic consumption in patients undergoing total knee arthroplasty. Orthop Surg 2017;9(01): $110-114$

8 Xu B, Ma J, Huang Q Huang ZY, Zhang SY, Pei FX. Two doses of lowdose perioperative dexamethasone improve the clinical outcome after total knee arthroplasty: a randomized controlled study. Knee Surg Sports Traumatol Arthrosc 2018;26(05):1549-1556

9 Kim JK, Ro DH, Lee HJ, Park JY, Han HS, Lee MC. Efficacy of systemic steroid use given one day after total knee arthroplasty for pain and nausea: a randomized controlled study. J Arthroplasty 2020; 35(01):69-75

10 Yu Y, Lin H, Wu Z, Xu P, Lei Z. Perioperative combined administration of tranexamic acid and dexamethasone in total knee arthroplasty-benefit versus harm? Medicine (Baltimore) 2019; 98(34):e15852

11 Lei Y-T, Xie J-W, Huang $Q$ et al. The antifibrinolytic and antiinflammatory effects of a high initial-dose tranexamic acid in total knee arthroplasty: a randomized controlled trial. Int Orthop 2019

12 Nadler SB, Hidalgo JH, Bloch T. Prediction of blood volume in normal human adults. Surgery 1962;51(02):224-232

13 Gross JB. Estimating allowable blood loss: corrected for dilution. Anesthesiology 1983;58(03):277-280

14 Kathariya R. Intra-operative hemorrhage: a review of literature. Journal of Medical Diagnostic Methods 2013;02(06):

15 Sehat KR, Evans RL, Newman JH. Hidden blood loss following hip and knee arthroplasty. Correct management of blood loss should take hidden loss into account. J Bone Joint Surg Br 2004;86(04): 561-565

16 Hall GM, Peerbhoy D, Shenkin A, Parker CJ, Salmon P. Relationship of the functional recovery after hip arthroplasty to the neuroen- docrine and inflammatory responses. Br J Anaesth 2001;87(04): 537-542

17 Wilhelm M, Ewers U, Schulz C. Revised and new reference values for some trace elements in blood and urine for human biomonitoring in environmental medicine. Int J Hyg Environ Health 2004; 207(01):69-73

18 Poeran J, Rasul R, Suzuki S, et al. Tranexamic acid use and postoperative outcomes in patients undergoing total hip or knee arthroplasty in the United States: retrospective analysis of effectiveness and safety. BMJ 2014;349:g4829

19 Kehlet H, Joshi GP. The systematic review/meta-analysis epidemic: a tale of glucocorticoid therapy in total knee arthroplasty. Anaesthesia 2019;75(07):856-860

20 Blanié A, Bellamy L, Rhayem Y, et al. Duration of postoperative fibrinolysis after total hip or knee replacement: a laboratory follow-up study. Thromb Res 2013;131(01):e6-e11

21 Zhang S, Xie J, Cao G, et al. Six-dose intravenous tranexamic acid regimen further inhibits postoperative fibrinolysis and reduces hidden blood loss following total knee arthroplasty. J Knee Surg 2019;34(02):224-232

22 Lei Y, Xie J, Huang Q, Huang W, Pei F. Additional benefits of multiple-dose tranexamic acid to anti-fibrinolysis and anti-inflammation in total knee arthroplasty: a randomized controlled trial. Arch Orthop Trauma Surg 2020;140(08):1087-1095

23 Ma J, Huang Z, Shen B, Pei F. Blood management of staged bilateral total knee arthroplasty in a single hospitalization period. J Orthop Surg Res 2014;9:116

24 Chen XX, Wang T, Li J, Kang H. Relationship between inflammatory response and estimated complication rate after total hip arthroplasty. Chin Med J (Engl) 2016;129(21):2546-2551

25 Kardash KJ, Sarrazin F, Tessler MJ, Velly AM. Single-dose dexamethasone reduces dynamic pain after total hip arthroplasty. Anesth Analg 2008;106(04):1253-1257table of contents.

26 Tammachote N, Kanitnate S. Intravenous dexamethasone injection reduces pain from 12 to 21 hours after total knee arthroplasty: a double-blind, randomized, placebo-controlled trial. J Arthroplasty 2020;35(02):394-400

27 Smith C, Erasmus PJ, Myburgh KH. Endocrine and immune effects of dexamethasone in unilateral total knee replacement. J Int Med Res 2006;34(06):603-611

28 Levy JH. Antifibrinolytic therapy: new data and new concepts. Lancet 2010;376(9734):3-4

$29 \mathrm{Wu}$ XD, Chen Y, Tian M, et al. Application of thrombelastography (TEG) for safety evaluation of tranexamic acid in primary total joint arthroplasty. J Orthop Surg Res 2019;14(01):214

30 Mao Y, Jin J, Daniel JL, Kunapuli SP. Regulation of plasmin-induced protease-activated receptor 4 activation in platelets. Platelets 2009;20(03):191-198

31 Fitch JC, Rollins S, Matis L, et al. Pharmacology and biological efficacy of a recombinant, humanized, single-chain antibody C5 complement inhibitor in patients undergoing coronary artery bypass graft surgery with cardiopulmonary bypass. Circulation 1999;100(25):2499-2506

32 Pinto A, Carnuccio R, Sorrentino R, Di Rosa M. The inhibition of platelet aggregation by activated macrophages is blocked by dexamethasone. Pharmacol Res 1993;27(02):165-172

33 Van Aelbrouck C, Jorquera-Vasquez S, Beukinga I, et al. Tranexamic acid decreases the magnitude of platelet dysfunction in aspirinfree patients undergoing cardiac surgery with cardiopulmonary bypass: a pilot study. Blood Coagul Fibrinolysis 2016;27(08): 855-861

34 Gill AM, Leiter EH, Powell JG, Chapman HD, Yen TT. Dexamethasone-induced hyperglycemia in obese Avy/a (viable yellow) female mice entails preferential induction of a hepatic estrogen sulfotransferase. Diabetes 1994;43(08):999-1004 
35 Waldron NH, Jones CA, Gan TJ, Allen TK, Habib AS. Impact of perioperative dexamethasone on postoperative analgesia and side-effects: systematic review and meta-analysis. $\mathrm{Br} \mathrm{J}$ Anaesth 2013;110(02):191-200

36 Nurok M, Cheng J, Romeo GR, Vecino SM, Fields KG, YaDeau JT. Dexamethasone and perioperative blood glucose in patients undergoing total joint arthroplasty: a retrospective study. J Clin Anesth 2017;37:116-122

37 Wainwright CL, Setji TL, Sata SD, et al. Development and implementation of a perioperative blood glucose monitoring protocol for patients undergoing spinal surgery. J Perianesth Nurs 2019;35 (02):135-139

38 Kehlet $\mathrm{H}$, Jensen TS, Woolf CJ. Persistent postsurgical pain: risk factors and prevention. Lancet 2006;367(9522):1618-1625
39 Safa B, Gollish J, Haslam L, McCartney CJ. Comparing the effects of single shot sciatic nerve block versus posterior capsule local anesthetic infiltration on analgesia and functional outcome after total knee arthroplasty: a prospective, randomized, double-blinded, controlled trial. J Arthroplasty 2014;29(06): 1149-1153

40 Shen Y, Guo Y, Mikus P, et al. Plasminogen is a key proinflammatory regulator that accelerates the healing of acute and diabetic wounds. Blood 2012;119(24):5879-5887

41 Polderman JAW, Farhang-Razi V, van Dieren S, et al. Adverse side-effects of dexamethasone in surgical patients - an abridged Cochrane systematic review. Anaesthesia 2019;74(07): 929-939 